\section{Military Technical College Kobry El-Kobbah, Cairo, Egypt}

\author{
$6^{\text {th }}$ International Conference \\ on Electrical Engineering \\ ICEENG 2008
}

\title{
Super capacitors as electrical power buffers in military use
}

\author{
By \\ Otakar Kurka* \\ Jan Leuchter*
}

\section{Abstract:}

The paper describes the possible sources of electrical energy for military purposes, deals with the necessity of electrical energy accumulation and gives brief analysis of different types of energy buffers, namely electrolytic capacitors, accumulators (batteries) and supercapacitors. Dynamical properties of developed electrical energy generation set with the variable speed-constant frequency technology have been analysed with respect to the effect of sudden extreme load increase. At low speeds the driving engine is overloaded and generator is not able to deliver sufficient peak energy. Supercapacitor as the controlled energy buffer in cooperation with respective DC/DC bi-directional converter and AC/DC/AC combined converter solves the problem. Results of measurements and verification of reliable system operation proving the credibility of performed analyses and calculations are discussed in the paper conclusions.

\section{Keywords:}

Electrical energy, sources, buffers, supercapacitors, generating sets

* University of Defence,

Faculty of Military Technology

Czech Republic 


\section{Introduction:}

Sources of electrical energy for military use are different with respect to the purpose, place, sort of appliance, type of supplied weapon systems. Fixed military facilities, bases and camps are supplied by common electrical network system. Uninterruptible power sources are used to span short network black-outs and to maintain the power quality supplied to computers and other sensitive electronic equipment due to dips, surges and voltage reductions.

In military vehicles and aircraft various types of accumulator batteries are used for starting of engines, on-board generators as main source of electrical energy are driven by operating engines.

Stable electrical power generating sets are used for power supply in military bases and camps, where the connection to network is not possible.

Mobile generating sets are used for general use to supply various appliances, facilities, weapon systems, for heating, illumination, and other purposes in military units. Most of up to date weapon systems are equipped by specialized built-in generating sets.

In studies focused on this problem the sources based on the small nuclear generators, sun and wind energy are speculated, but no one from these technologies is suitable from the mobility, camouflage, safety and operativity of corresponding application point of view. The majority of above mentioned electrical energy sources use some means of energy accumulation (electrical energy buffers or accumulators) to secure the reliable operation under all possible circumstances and conditions.

It is considered as self-evident that fuel cells become another mobile power sources and electrical energy buffers (accumulators). Fuel cells were marked as one of the new energetic source alternatives for military applications. In the comparison with classical conversion of fuel (where the efficiency reaches 15 to $30 \%$, and with the gas turbine with max. 40\%), fuel cells based on the direct conversion of chemical energy to the electric one reach efficiency 50 to $80 \%$, according to the type, power, used chemicals and design. In the combination with semiconductor converters, systems based on the fuel cells become practically the universal source of electric energy.

\section{Accumulation of electrical energy:}

With the development of new technologies in transportation, vehicles, renewable energy sources, UPS, mobile electrical energy generating sets and in other branches the accumulation of electrical energy, its transformation and transportation represents one common problem. For this purposes various types of electrical energy buffering methods and converters including buffers are used. As will be shown, supercapacitors 
are well suited to replace classical batteries and conventional capacitors in many applications.

Vehicle electrification can lead to significant improvements in vehicle performance, energy conservation and polluting emissions. The problem of on-road electrical and hybrid vehicles is described in many articles, discussed on many conferences and solved by many vehicles and automobiles developing and producing companies. Supercapacitors have replaced or are being tested to replace batteries in engine starting applications such as large diesel generators, military tank engines, submarine and locomotive engines. For these applications ultracapacitors take up less space and weight than conventional batteries, but posses excellent cold weather capabilities for starting.

Compared to on-road vehicles, off-road and namely military vehicles have some specific requirements, such as high hill climbing ability, obstacle negotiation, crosscountry travel, hard acceleration, high speed, silent sneak operation, silent watch, low signature and others. To meet these requirements, the vehicle must have the power sources generating very low noise while operating, such as electric motor, and corresponding energy supply, such as batteries and supercapacitors. Among various technologies, hybrid drivetrain consisting of internal combustion engine, electric motors and energy accumulators (supercapacitors) are most suitable to meet requirements for necessary electrical energy accumulation.

Renewable electrical energy sources, namely wind and solar power plants are dependent on wind and sun. For the time of windlessness and darkness the part of the produced energy must be accumulated. One of possibilities of produced energy accumulation is its transformation to hydrogen and oxygen by means of water electrolysis. $\mathrm{H}_{2}$ and $\mathrm{O}_{2}$ can be then used in fuel cells for the delivery of electrical energy independently on the weather conditions. Newertheles, namely in smaller, on the network independent wind and sun powerplants the electrical energy is accumulated in chemical accumulators and supercapacitors.

In uninterruptible power sources the supercapacitors substitute hitherto used chemical accumulators. UPCs maintain the power quality supplied to computers and other sensitive electronic equipment due to black-outs, dips, surges and voltage reductions. Load leveling is achieved by absorbing power during surges and discharging power during dips.

The basic instrument of modern electromagnetic weapons is the powerful energy buffer with the ability to deliver extremely high power peak in short time. Such electromagnetic weapons as DEWL (Directed Energy Weapons Lasers), PBW (Particle Beam Weapons), DEWRF (Directed Energy Weapon Radiofrequency), DEWM (Directed Energy Weapons Microwave) are based on the effect of huge amount of electrical energy accumulation and very short time discharge in the form of power pulses. In spite of some limitations, even in this field the supercapacitors find their indispensable role. 
Mobile electrical energy generating sets (EGS) still are and will probably be in near future based mainly on generators driven by combustion engines using fossil fuels. In order to increase efficiency, decrease the fuel consumption and optimize the operational conditions of mobile electrical power generating sets, the VSCF (variable speedconstant frequency) technology is used. Variable output voltage and frequency are transformed to constant values by means of power electronics converters. Due to unconvenient dynamical properties of VSCF based EGS the electrical energy buffers (accumulators) create the essential part of the system securing its reliable operation.

\section{The comparison of electrical energy buffers:}

As an example let us analyze the possibility to store and deliver the energy peak necessary to overlap the energy shortage lasting 2 seconds in EGS with VSCF technology with the power output $6 \mathrm{~kW}$ discused later in this paper. As mentioned above three different configurations of energy storage unit can be hitherto used: electrolytic capacitor, accumulator (battery) and supercapacitor. This configuration with different energy storage can have same advantageous or disadvantageous properties that are compared in the following text.

Energy buffers can be solved by means of electrolytic capacitors but energy of $12 \mathrm{~kJ}$, which is to be delivered during 2 second by $6 \mathrm{~kW}$ EGS (see eq. 8), is too high for this solution. Electrolytic capacitor itself can accumulate comparatively low energy. The eq. 1 shows resultant capacity value to create energy bank $12 \mathrm{~kJ}$ during $2 \mathrm{~s}$.

$C=\frac{\Delta t}{\Delta U} \cdot I_{d}=\frac{\Delta t}{\Delta U} \cdot \frac{P}{U_{d}}=\frac{2}{50} \cdot \frac{6000}{600}=0.4 F$

To obtain the figure about the costs of the electrolytic capacitor: Jamicon E2200M/385V cost about 100 Euro. Dimensions of this capacitor are $75 \times 105 \mathrm{~mm}$ and weight $250 \mathrm{~g}$. Necessary number of capacitors is given by eq. 2. Solution of energy buffers with 364 capacitors brings radical increase of weight and price.

$x=\frac{C_{\text {total }}}{C_{\text {cell }}} \cdot \frac{U_{\text {no } \min a l}}{U_{\text {cell }}}=\frac{0.4 F}{2200 e^{-6} \mathrm{~F}} \cdot \frac{800 \mathrm{~V}}{400 \mathrm{~V}}=364[-]$

Solution of energy buffers by means of accumulator (battery) can bring a lot of storage energy. E.g. accumulator LONG (output voltage $12 \mathrm{~V}$; capacity $7.2 \mathrm{Ah}$; dimensions $151 \times 65 \times 94 \mathrm{~mm}$; weight $2.68 \mathrm{~kg}$; price 11 Euro) can deliver $6 \mathrm{~kW}$ energy with current $125 \mathrm{~A}$ during $10 \mathrm{~s}$. The load characteristics of accumulators discharging are shown in Fig. 1. Energy of accumulator can be expressed by equation (3). One of curves (y) of output characteristics from Fig. 1 was approximated by quadratic polynom: 
$y=-0.0000012 t^{2}+0.0006 t+12.629$

and by substitution to the equation (4) the quantity of energy stored is about $160 \mathrm{~kJ}$ :

$$
\begin{aligned}
& W=Q \cdot U=\int_{0}^{t} u(t) \cdot I d t=I \cdot \int_{0}^{t} u(t) d t= \\
& 7 \int_{0}^{1800}\left(-0.0000012 \mathrm{t}^{2}+0.0006 \mathrm{t}+12.629\right) d t \approx 160 \mathrm{~kJ}
\end{aligned}
$$

For example: $12 \mathrm{~V}$ and 7.2 Ah lead acid accumulator batteries connected in series with output voltage $48 \mathrm{~V}(4 \times 12 \mathrm{~V})$ and DC/DC converter with output voltage $600 \mathrm{VDC}$ can accumulate energy cca $640 \mathrm{~kJ}$, see Fig. 1, that is much more than was required $(12 \mathrm{~kJ})$.

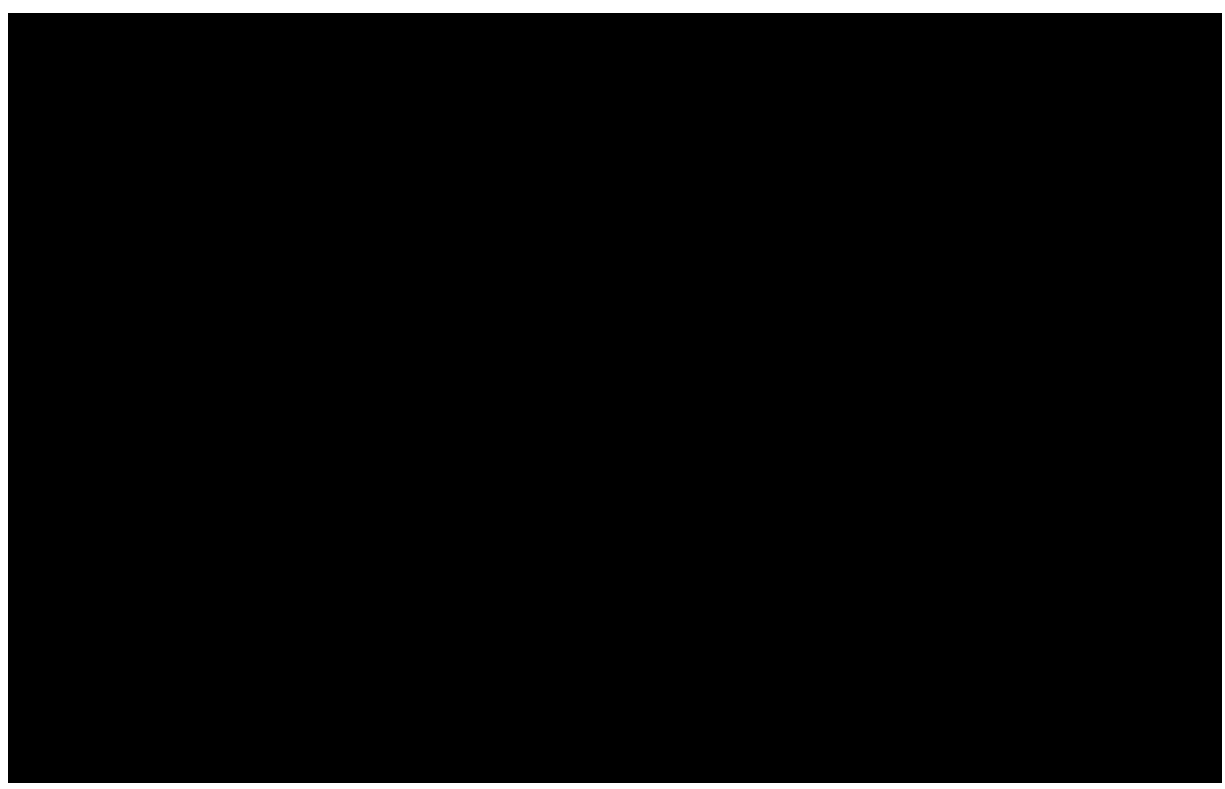

Figure (1): Discharging of accumulator LONG [9]

High current that is not friendly for operating time of accumulator can be decreased by means of voltage increasing e.g. by series connection of higher number of accumulators $(25 \times 12 \mathrm{~V})$. This solution can bring with the same type of accumulators (LONG) the energy about $4 \mathrm{~kJ}$ available for 8 minutes with discharge current $20 \mathrm{~A}$. For lower power output EGS with accumulator bank can operate without diesel engine running more than 1 hour. That can be useful during military action (without noise and harmful emissions). Solution of EGS with accumulators brings for both concept of voltage proposals weight increasing of EGS as much as $60 \mathrm{~kg}$. 
Super-capacitors are the further solution of buffer problem. They found their place in many applications and are opening new area of energy accumulation. There are combined advantageous features in comparison to previous technologies of energy storage. The supercapacitors can store much more energy then electrolytic capacitors. Present supercapacitors are readily available with capacity $3500 \mathrm{~F}$. Usually, the accumulators have limitation in number of life cycles. Supercapacitors, on the other hand, can be charged and discharged almost unlimited number of times.

Energy of electrical field of capacitors can be expressed by universal equation (5) including the fact that supercapacitors are not discharged fully but only to half of nominal voltage $\mathrm{U}_{\text {nom }}$.

$$
W=\frac{1}{2} \cdot C \cdot \Delta U^{2}=\frac{1}{2} \cdot C \cdot\left(U_{n o m}^{2}-\left(\frac{U_{n o m}}{2}\right)^{2}\right)
$$

The resultant required capacity that can store energy $12 \mathrm{~kJ}$ for peak energy delivery at nominal voltage e.g. $100 \mathrm{~V}$ of supercapacitors is given by formula (6). Final capacity $3.2 \mathrm{~F}$ is comparatively high but realization by means of supercapicitors for the present time is possible.

$$
C=\frac{2 \cdot W}{\left(U_{n o m}^{2}-\left(\frac{U_{n o m}}{2}\right)^{2}\right)}=\frac{2 \cdot 12 k}{100^{2}-\left(\frac{100}{2}\right)^{2}} \approx 3.2 F
$$

In Fig. 2 results of effect of nominal voltage of supercapacitors versus number of capacitors (price) and average current are shown in dependence on time of delivery 6 $\mathrm{kW}$ power to the load and on the average current delivered by the supercapacitor.

Every dependence parameters on the nominal voltage of supercapacitors are very important for supercapacitors bank design. Modules BPAK0350-15 Maxwell have been chosen. Their parameters are: $58 \mathrm{~F}$; $15 \mathrm{~V}$; DC resistance $19 \mathrm{~m} \Omega$; dimensions 216x69x38 $\mathrm{mm}$; weight $0.5 \mathrm{~kg}$; price about 210 Euro. From fig. 2 the optimum value of nominal voltage $100 \mathrm{~V}$ can be determined. The analysis mentioned above show that the nominal voltage $100 \mathrm{~V}$ can by obtained by series configuration of 7 modules of BPAK0350-15. This module can use high charge and discharge current for very quick delivery of peak energy to the load. This is very important for EGS concept with separated energy buffer for peak energy delivery. This concept can store about $34 \mathrm{~kJ}$ according to the formula (7) and can deliver $6 \mathrm{~kW}$ during 5 seconds sufficient for EGS reliable operation. 


$$
\begin{aligned}
W & =\frac{1}{2} C \cdot\left(U_{\text {nom }}^{2}-\left(\frac{U_{\text {nom }}}{2}\right)^{2}\right)=\frac{1}{2} \cdot\left(C_{\text {cell }} \cdot \frac{\# \text { parallel }}{\text { \# series }}\right) \cdot\left(U_{\text {nom }}^{2}-\left(\frac{U_{\text {nom }}}{2}\right)^{2}\right)= \\
& =\frac{1}{2} \cdot\left(58 \cdot \frac{1}{7}\right) \cdot\left(105^{2}-\left(\frac{105}{2}\right)^{2}\right)=34.3 \mathrm{~kJ}
\end{aligned}
$$

The main disadvantage of high nominal voltage is high internal resistance $\mathrm{R}_{\mathrm{i}}$. Internal resistance of single module is $0.002 \Omega$. Resistance of seven modules is $0.014 \Omega$. In comparison with series combination of 25 accumulators, where the internal resistance is $25 \times 0.06 \Omega=1.5 \Omega$, this value seems to be negligible. Nevertheless this internal resistance decline the gain and higher internal resistance decreases substantially efficiency of energy storage.

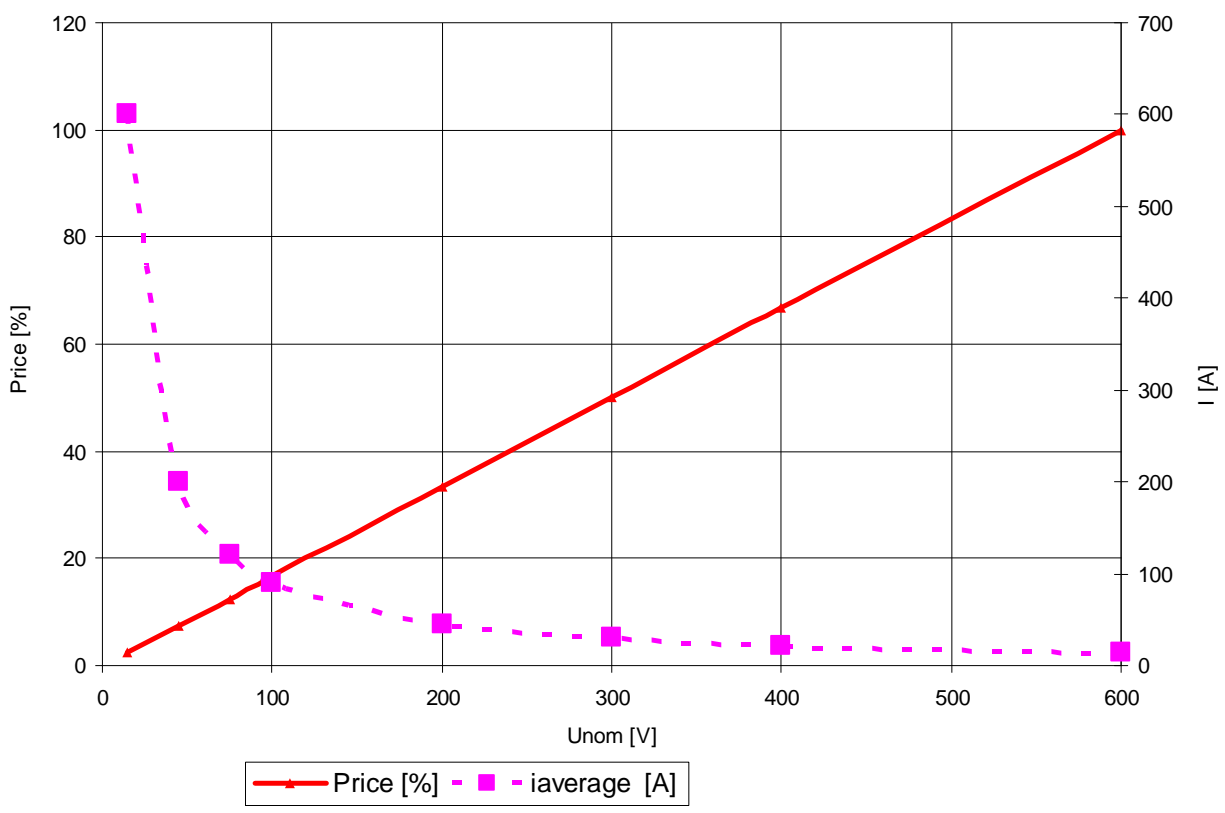

Figure (2): Optimalisation of nominal voltage design of supercapacitors bank

Solution of storage unit with electrolytic capacitors is the worst in the all aspects. Much more interesting are solutions using battery and supercapacitors. Figure 3 ilustrates the comparison of different energy storage battery (Lead-Acid, Li-Ion, Ni-Mh, $\mathrm{Ni}-\mathrm{Cd}$ and Supercaps units) in relation to the price, weight and value of stored energy. Accumulators can bring very good relationship between stored energy and price. Accumulator storage unit however brings weight increasing of EGS as much as $60 \mathrm{~kg}$. Supercapacitors bring better relationship between weight and price. 


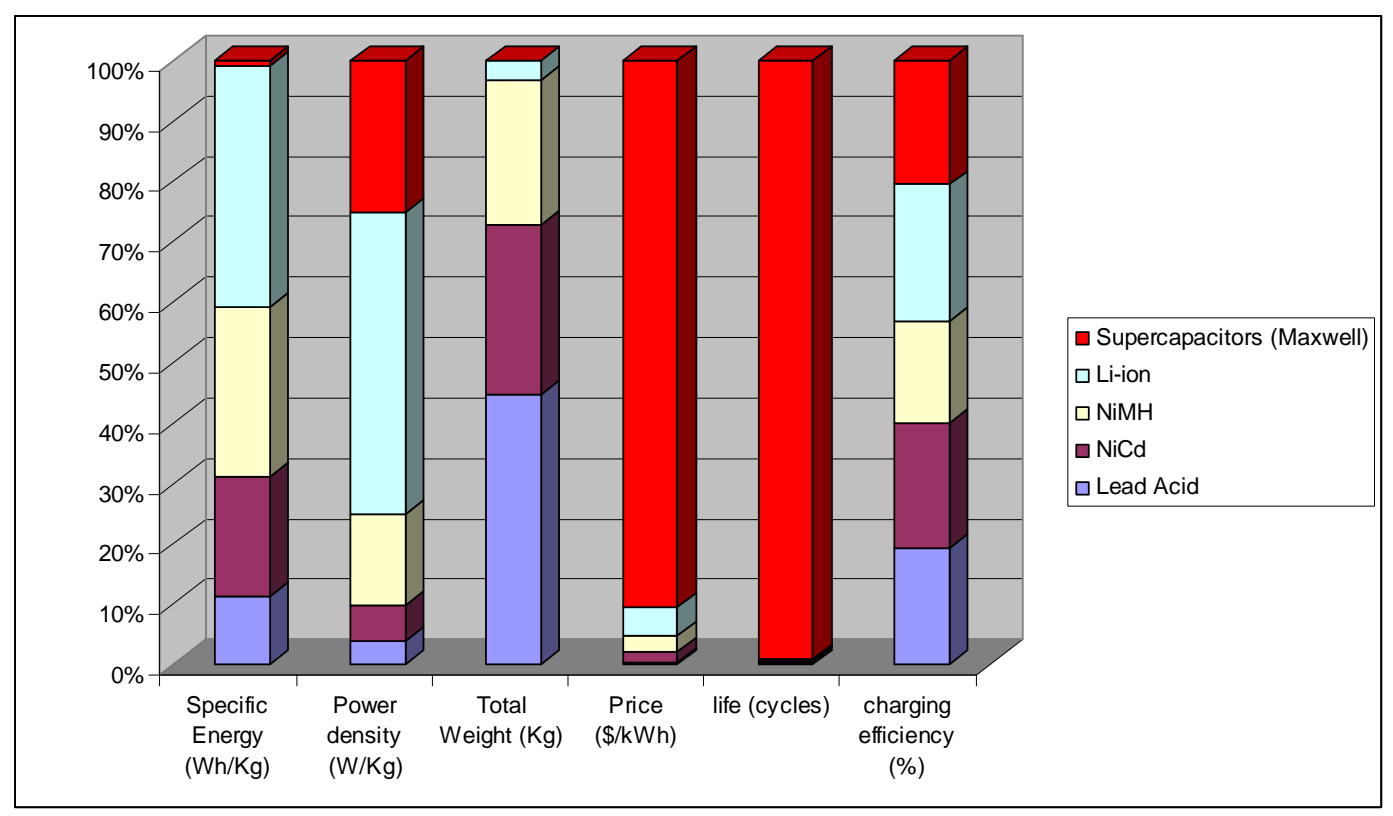

Figure (3): Comparison of energy buffers [10]

Generally it can be stated that supercapacitors can be charged and discharged almost an unlimited number of times, can be charged and discharged in terms of miliseconds, seconds or minutes, have very high power density, do not release any thermal heat during discharge due to negligible internal resistence, cannot be overcharged, are not affected by deep discharges as are chemical batteries, have a long lifetime (80\% of capacity after 10 years, lifetime up to 20 years), the DC/DC round trip efficiency $80 \%$ to $95 \%$ in most applications, operating temperature range between $-50 \mathrm{C}$ and $85 \mathrm{C}$, are environment friendly.

Figure (4): The system EGS with optimum variable speed of engine 


\section{The new generation of EGS with supercapacitors as energy buffers:}

The new generation of EGS under the development is based on the variable speed concept, see Figure 4. The diesel engine changes the speed according to the load of the set. The optimum speed is hereby calculated according to the load of the EGS with the optimality criterion of minimum fuel consumption. The analysis of the dynamic behavior of such new EGS concept indicates some problems with power and voltage drops at sudden increase of the load. The dynamical properties of EGS concept with variable speed technology could be improved by using an energy buffer. The following result of experiments (Fig. 5) show the oscilloscopic records of dynamic behavior of the EGS with optimum variable speed of engine. The yellow curve $(\mathrm{CH} 1)$ represents current of AC/DC rectifier, the red curve $(\mathrm{CH} 4)$ shows engine speed. The output power is calculated from output DC voltage and current. The result of calculation is shown as violet curve (MATH).

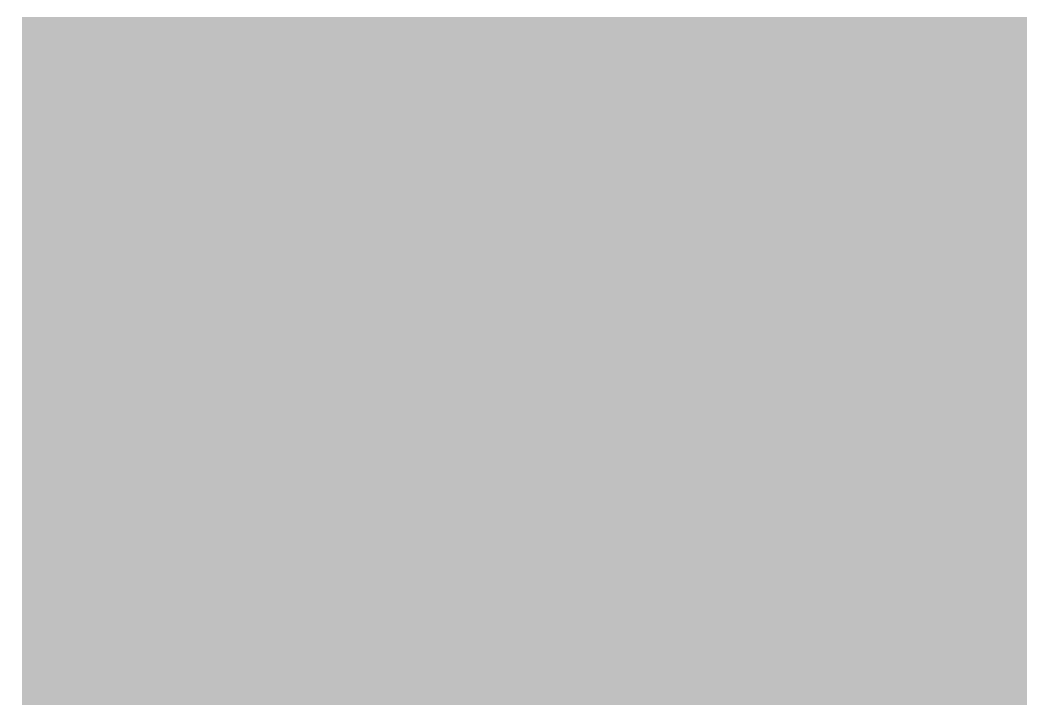

Figure (5): The load change of EGS from 0.6 to $6 \mathrm{~kW}$ at $1460 \mathrm{rpm}$

The load change from low load to higher load is given by point $\mathrm{A}$ in the figure. In time period $t_{1}$ system was loaded by power $448 \mathrm{~W}$ at the speed of $1460 \mathrm{rpm}$. In time point A the load was changed from $448 \mathrm{~W}$ to $5600 \mathrm{~W}$. During the time $\mathrm{t}_{2}$ the transient process is in progress. Time period $t_{3}$ shows steady state of the system. As can be seen, the sudden power load increase can stop the diesel engine. The engine is in this case not able to produce sufficient torque and EGS generator cannot deliver required energy to the load. The diesel engine cannot operate with optimum speed for every load and there is not enough power for every at every optimum speed of engine. EGS must be accomplished with electrical energy buffer similar to UPC system. 
Energy buffer can improve dynamic behavior of VSCF based EGS at sudden transients from low load to high load by means of inserting accumulated energy to the voltage link of the DC/DC converter, as shown in Fig.6. This concept is based on the delivery of peak power from energy buffer to the link capacitor of DC/DC converter during the change of engine speeds.

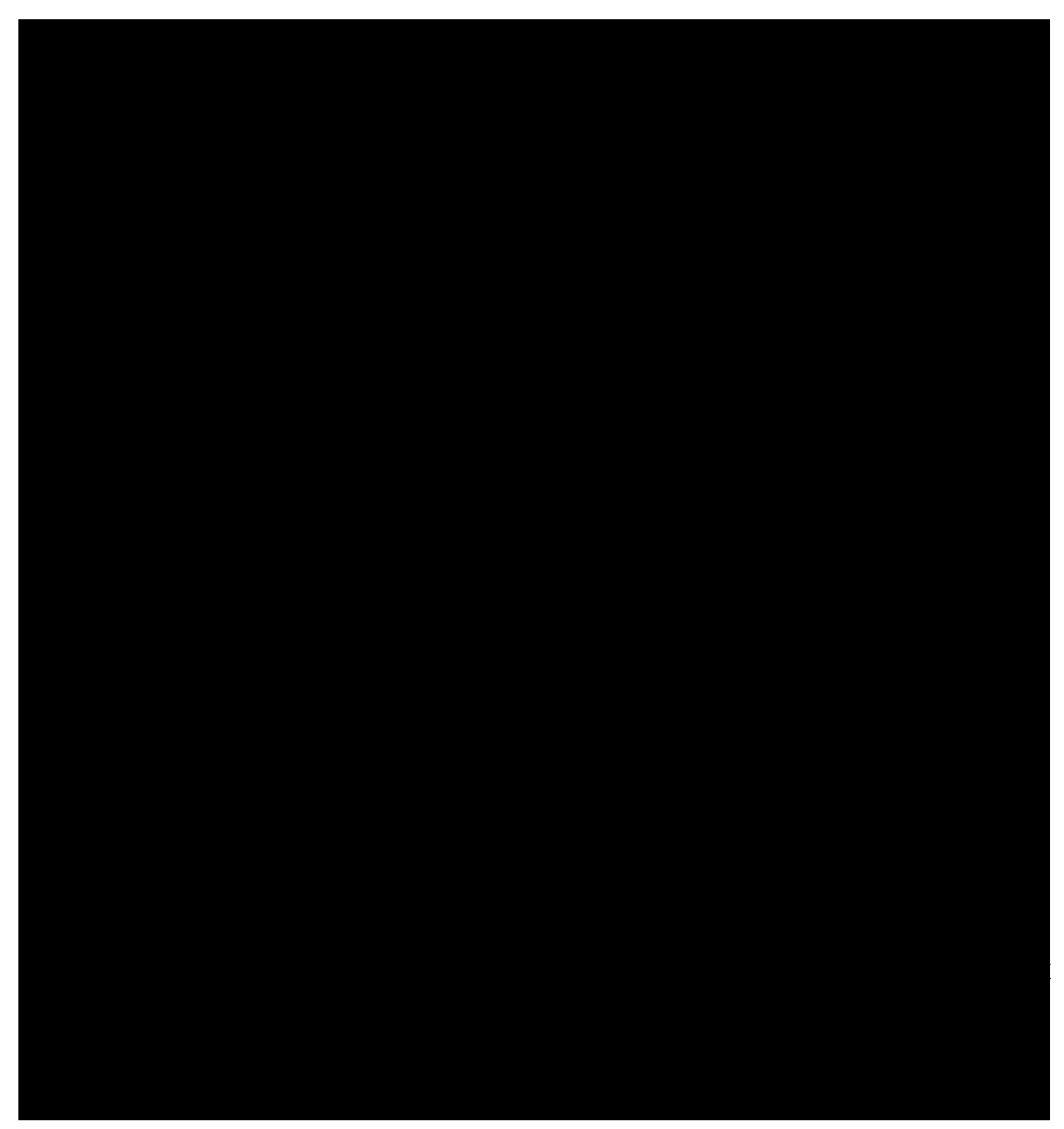

Figure (6): The VSCF based EGS with energy buffer

The requested energy $\mathrm{W}$ is given by maximal required power $\mathrm{P}$ and average time of the regulation $\mathrm{T}_{\text {Reg. }}$. According to the measurements of dynamic behavior it is necessary to cover the energy peak of $6 \mathrm{~kW}$ during approximately $2 \mathrm{~s}$, see Eq. 8.

$W=P \cdot T_{\operatorname{Re} g} \notin 6000 \cdot 2=12 \mathrm{~kJ}$

According to Eq. 8 the system of EGS with energy buffer must include extra energy of $12 \mathrm{~kJ}$ for overlap of peak power during speed change. As mentioned above, energy 
buffer can be feasible with electrolytic capacitors, but $12 \mathrm{~kJ}$ of energy is too high. Series connection of electrolytic capacitors products high cost of such energy buffer. Advantage electrolytic capacitors are easy insertion directly to the link without additional power bi-directional converter. Solution of energy buffer with accumulator battery can bring enough energy storage (much more than $12 \mathrm{~kJ}$ ), but on the other side accumulators bring enormous EGS weight increase. Energy buffer with supercapacitors can bring ideal solution due to much higher charging and discharging efficiency in comparison to accumulators. Supercapacitors with nominal voltage $100 \mathrm{~V}$ and capacity $3.2 \mathrm{~F}$ can store and deliver sufficient energy $12 \mathrm{~kJ}$ to boost up EGS output power during the assumed sudden load increase at low EGS speed.

Output variable AC voltage of generator, corresponding to the variable engine speed, must be converted to the constant AC voltage by means of power electronics, see fig. 6 . Output voltage of generator is rectified by means of three-phase AC/DC diode rectifier. If the output voltage of a three-phase diode rectifier is less than $570 \mathrm{~V}$, than DC/DC converter increases the voltage to the required value for DC/AC converter transforming the DC voltage to the constant three-phase AC voltage $(400 \mathrm{~V} / 50 \mathrm{~Hz})$. In Fig. 6 two possibilities (A or B) of using a bi-directional converter with supercapacitors in the DC link of $\mathrm{AC} / \mathrm{DC} / \mathrm{AC}$ converter are shown. The fist connection (B) requires DC link of rectifier and the second (A) requires DC link of DC/DC. Connection (B) requires lower value of output voltage of bi-directional converter. This can be an advantage, because the cost of converter can be lower in comparison with solution A. The connection (A) requires always $570 \mathrm{~V}$ on the output of bi-directional converter.

Figure (7): The verification of VSCF based EGS concept with energy buffer 
The function of the buffer power peak delivery in EGS has been experimentally verified. Results shown in Fig. 7. satisfy the conditions and expentancy. The yellow curve $(\mathrm{CH} 1)$ is the same as in Fig. 5 and shows current of rectifier. The red curve $(\mathrm{CH}$ 4) represents engine speed. Output DC voltage is depicted by green curve $(\mathrm{CH} 2)$. The output power calculated from output DC voltage and DC current is presented by violet curve (MATH). In time $t_{1}$ system was loaded by load power $1 \mathrm{~kW}$ at the speed of 1700 $\mathrm{rpm}$. In time $\mathrm{t}_{2}$ the power load was changed from $1 \mathrm{~kW}$ to $4.5 \mathrm{~kW}$. During this time $(1.24 \mathrm{~s})$ the transient process is in progress and power is delivered from energy buffer. Time $t_{3}$ shows steady state of system. As can be seen, the system accepted the change of power load and feed-back control of engine speed adjusted an optimal speed of engine. And so, concept EGS with variable speed of engine can be applied.

\section{Conclusions:}

Possible sources of electrical energy for military purposes and the necessity of electrical energy accumulation have been discussed with brief analysis of different types of energy buffers, namely electrolytic capacitors, accumulators (batteries) and supercapacitors.

The analysis of developed mobile electrical power source based on VSCF technology proved, that dynamic behavior of diesel engine can affect unfavourably the output electrical energy quality.

Electronic converter can improve the dynamic behavior of whole system by means of inserting accumulated energy to the $\mathrm{DC}$ link of $\mathrm{AC} / \mathrm{DC} / \mathrm{AC}$ converter. As an energy buffer supercapacitors can be used.

The detail analysis of energy buffers system variations shows, that bi-directional converters used in the storage system bring high efficiency in reference to price.

The experimental verification of above described solutions proves the credibility of performed analyses and calculations. 


\section{References:}

[1] L.M. Tolbert, W.A. Peterson, C.P. White, T.J. Theiss, and M.B. Scudiere, "A bidirectional dc-dc converter with minimum energy storage elements", $\mathrm{P}$ in Conf. Rec. IEEE IAS Annu, pp. 1572-1577, 2002.

[2] H. Knitterscheidt, "Neue Generation SEA füer militärische Nutzung, " Symposium moderne elektrische Energietechnik, Germany, 1998, pp. 14.1 - 14.22.

[3] Marco Piemontesi, GE Digital Energy Riazzino, "Alternative Energy Storage Systems for UPS, “ PCIM 2006, Nuremberg-Germany.

[4] W. Koczara, N. Al-Khayat, R. Seliga, J. Al-Tayie, "Variable speed integrated generating set an emerging technology for distributed power generation," Power Tech Conference Proceedings, vol. 3, 2003.

[5] J. Leuchter, V. Reřucha, Z. Krupka, P. Bauer. Dynamic Behavior of Mobile Generator Set with Variable Speed and Diesel Engine. In Proceedings of the 38th Annual IEEE Power Electronics Specialist Conference (PESC 2007), Orlando : PESC Press, 2007, p. 2287 - 2293. ISBN 1-4244-0655-2.

[6] J. Leuchter, O. Kurka, P. Bauer, V. Řeřucha. Modelling and Experimental Verification of Mobile Power Sources with Variable Speed, Journal of Electrical Engineering (JEE EC). September/October 2006, vol. 57, no. 5, p. 241-248. ISSN 1335-3632.

[7] J. Moreno, M. E. Ortuzar, J. W. Dixon, "Energy-management system for a hybrid electric vehicle, using ultracapacitors and neural networks, "IEEE Transactions on Industrial Electronics, vol. 53, Issue 2, 2006, pp. 614 - 623.

[8] J. Leuchter, P. Bauer, V. Steklý. "System Variation of Electrical GEN-SET with Energy Buffer, "In Proceedings of the 4th Power Conversion Conference (PCC Nagoya 2007), Nagoya : PCC Press, 2007, p. 1401 - 1406. ISBN 1-4244-0844-X and IEEE Catalog Number 07TH8935.

[9] http://www.klb.com.tw

[10] J. Leuchter, P. Bauer. Efficiency Investigation of Electrical Generator-Converter Set. IEEJ Transaction on Industry Applications, November 2007, vol. 127, no. 10, p. 1064-1069. ISSN 0913-6339.

\section{Acknowledgements:}

The research work is supported by the Czech Ministry of Education, Youth and Sports (project no. OC169/COST542) and Reserch program No VZ-0000403. 